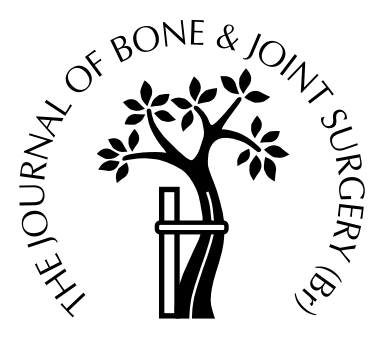

\title{
Ultrasound in the management of the position of the femoral head during treatment in a spica cast after reduction of hip dislocation in developmental dysplasia of the hip
}

Florens Q. M. P. van Douveren, Hans E. H. Pruijs, Ralph J. B. Sakkers,

Rutger A. J. Nievelstein, Frederik J. A. Beek

From the Wilhelmina Children's Hospital, Utrecht, The Netherlands

$\mathbf{I}^{\mathrm{n}}$ n this prospective study of $\mathbf{1 8}$ hips we compared the efficacy of ultrasound with $\mathrm{CT}$ in determining the position of the femoral head in a spica cast after closed or open reduction in children with developmental dysplasia of the hip. Ultrasound was performed through the perineal opening of the cast. With a transinguinal approach, the superior ramus of the pubis, the acetabulum, the femoral head and the femoral neck can be depicted in one plane. The CT and ultrasound images were blinded and reviewed by two of the authors.

Ultrasound was inconclusive in the first two reductions since the perineal opening was too small to see all the landmarks in one plane. In the following 16 reductions the landmarks were well defined and interpretation of the CT and ultrasound was similar. The perineal opening in the spica cast should be made in such a way that the ultrasound probe can be positioned in the groin so that the landmarks can be shown in one plane.

J Bone Joint Surg [Br] 2003;85-B:117-20.

Received 23 July 2001; Accepted after revision 7 December 2001

In children with developmental dysplasia of the hip the exact position of the femoral head in a spica cast after closed or open reduction of a dislocated hip is often difficult to assess on standard radiographs. The density of the casting material can impair clarity. Only the ossified portion of the femoral head is seen on radiographs. The two-dimensional properties of the image do not allow accurate assessment of the position of the femoral head in the anteroposterior (AP) plane. ${ }^{1}$

F. Q. M. P. van Douveren, MD, Orthopaedic Surgeon

H. E. H. Pruijs, MD, PhD, Orthopaedic Surgeon

R. J. B. Sakkers, MD, PhD, Orthopaedic Surgeon

Department of Pediatric Orthopaedic Surgery

R. A. J. Nievelstein, MD, PhD, Radiologist

F. J. A. Beek, MD, PhD, Radiologist

Department of Radiology

Wilhelmina Children's Hospital, UMC Utrecht, Lundlaan 6, 3584 EA Utrecht, The Netherlands.

Correspondence should be sent to Dr H. E. H. Pruijs.

(C)2003 British Editorial Society of Bone and Joint Surgery

doi:10.1302/0301-620X.85B1.12665\$2.00
At present $\mathrm{CT}$ is the preferred method for determining the position of the femoral head in the AP plane in a spica cast after closed or open reduction. ${ }^{2-4}$ Smith et $\mathrm{al}^{3}$ reported that the use of the modified Shenton line was the most accurate method of determining the location of the femoral head. This is a line drawn parallel to the anterior aspect of the pubic rami, which should cross the antero-medial edge of the femoral metaphysis. A low intra- and interobserver variability has been found when using this method. ${ }^{3,5}$ Ultrasound is widely used to assess the position of the hip during treatment in a Pavlik harness. ${ }^{6-12}$ Our aim was to ascertain whether ultrasound was a satisfactory method of determining the position of the femoral head in relation to the acetabulum in a spica cast after closed or open reduction.

Patients and Methods

Between January 1998 and January 2000 we performed a prospective study on 13 children with developmental dysplasia of the hip. Approval had been given by the Medical Ethics Committee. There were eight girls and five boys. The right side was affected in six hips and the left in nine. The mean age at diagnosis was eight months (1 to 22) and at reduction 15 months (10 to 26). Two children had bilateral and 11 a unilateral dislocation of the hip. We initially treated 11 hips by closed reduction and four by primary open reduction. Two of the 11 hips remained unstable after closed reduction and had a secondary open reduction. In one, a posterior subluxation was seen after open reduction and a new spica cast was applied after repositioning the hip. A total of 18 reductions was studied.

In all patients, CT and ultrasound were used to check the position of the femoral head in the spica cast. For CT we used a Philips Tomoscan EVT, using a spiral scanning mode with a collimation width of $3 \mathrm{~mm}$, a pitch of 1 and a reconstruction index of $2 \mathrm{~mm}$. The scanned area was determined from a scout view, with the intention of examining from the upper rim of the acetabulum to the inferior aspect of the femoral epiphysis (Fig. 1).

Ultrasound examination was carried out immediately after CT on the CT table using an ALOKA SSD 1700 machine with a $7.5 \mathrm{MHz}$ convex probe. A transinguinal approach in the transverse plane through the perineal opening of the spica cast was used (Fig. 2). The perineal opening 


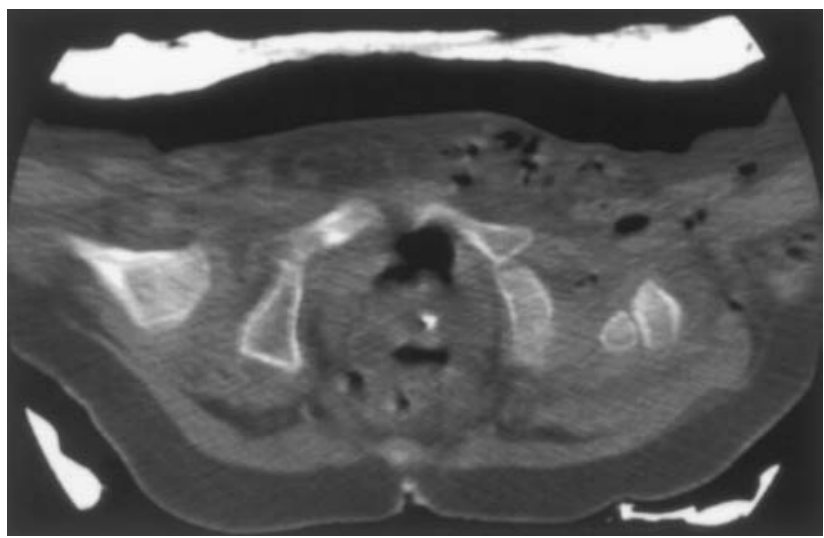

Fig. 1a
$\mathrm{R}$

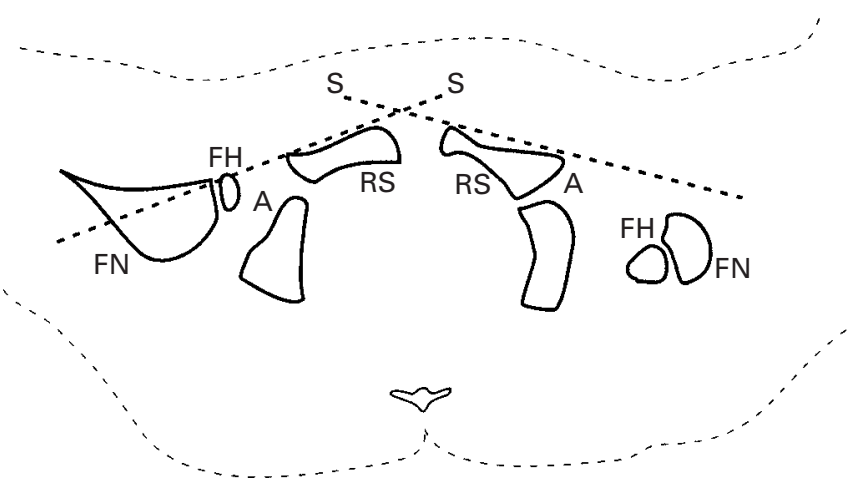

Fig. 1b

CT scan (a) and diagram (b) showing adequate reduction on the right side, but a persistent posterior dislocation on the left side, in a two-year-old girl with bilateral dislocated hips. (L, left; R, right; RS, ramus superior of the pubic bone; A, acetabulum; FH, femoral head; FN, femoral neck; s, Shenton line modified according to Smith et $\mathrm{al}^{3}$ )

Table I. Final diagnosis by ultrasound and CT in the 16 hips suitable for analysis after reduction

\begin{tabular}{lrlr}
\hline & CT & \\
\cline { 2 - 3 } & Reduced & Dislocated & Total \\
\hline Ultrasound & & 0 & \\
Reduced & 14 & 2 & 14 \\
Dislocated & 0 & 2 & 2 \\
Total & 14 & & 16 \\
\hline
\end{tabular}

had to be sufficiently large to allow adequate scanning by the ultrasound probe. The $7.5 \mathrm{MHz}$ probe was $3 \mathrm{~cm}$ in diameter and therefore suitable for a small scan area. It had a convex profile which made it easier to scan in an area with an irregular surface. The scan plane transected the superior ramus of the pubis, the acetabulum, the femoral head and the femoral neck in one plane (Fig. 3). The ultrasound examination was undertaken by a radiologist who did not know the result of the CT scan.

Both the CT and ultrasound images were blinded and reviewed by two of the authors, a radiologist and an orthopaedic surgeon. On both the CT scan and ultrasound the reduction was determined according to the modified Shenton line. ${ }^{3}$

\section{Results}

In the first two hips the position of the femoral head was not seen clearly on the ultrasound images. These were therefore considered to be unsatisfactory because the landmarks were not depicted in one scan plane. They were the first in the series and the perineal opening in the spica cast was too small to allow an adequate ultrasound investigation. They were therefore excluded from further analysis.

The interpretation of the ultrasound and the CT scans was the same in the remaining 16 investigations. Ten of 12

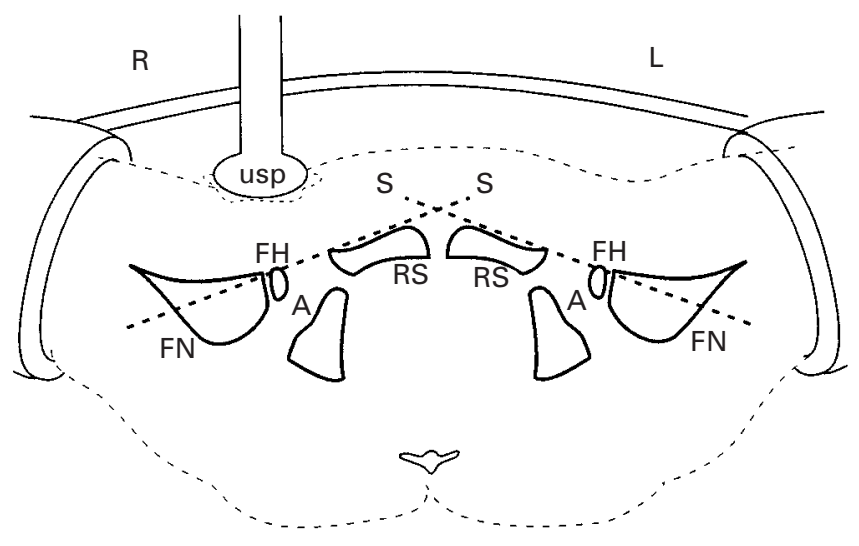

Fig. 2

Diagram showing how the ultrasound investigation is done through the perineal opening of the spica cast. Scanning is by a transinguinal approach in a transverse plane (usp, ultrasound probe; L, left; R, right; RS, ramus superior of the pubic bone; A, acetabulum; FH, femoral head; FN, femoral neck; s, Shenton line modified according to Smith et $\mathrm{al}^{3}$ ).

closed reductions and four of six open reductions showed an adequate position of the femoral head on ultrasound and CT. In two, a persistent dislocation was seen on ultrasound and CT scan (Table I).

\section{Discussion}

In order to reduce the amount of ionising radiation during the treatment of hip dislocation in children with developmental dysplasia of the hip, we investigated the use of ultrasound after reduction and in the spica cast. Ultrasound is easy to use in the operating theatre and in the outpatient department unlike MRI.

Axial CT which is considered to be the method of choice for visualisation of the position of the femoral head 


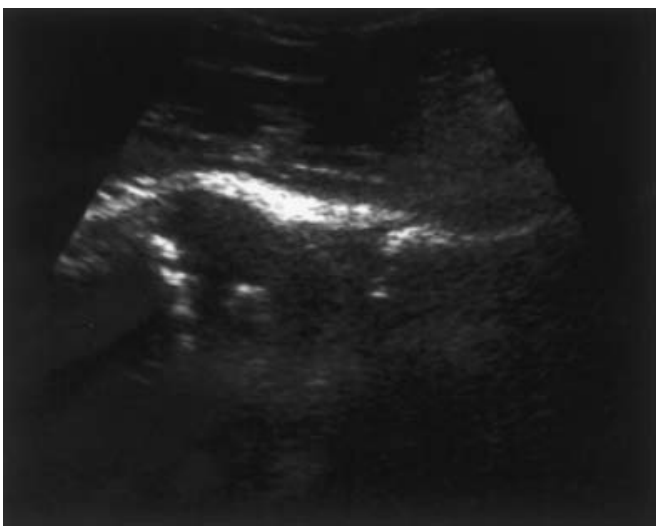

Fig. 3a

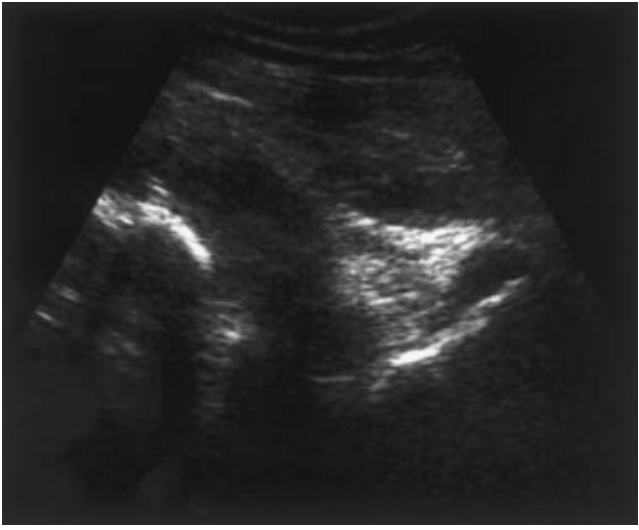

Fig. 3c

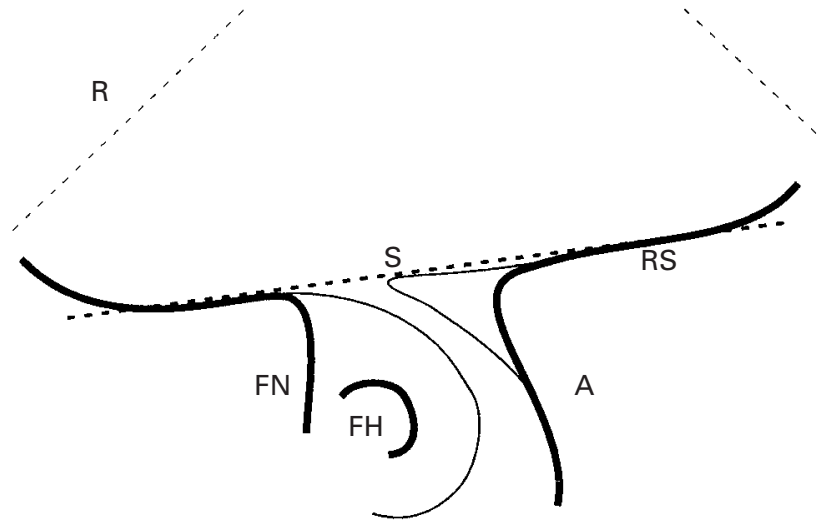

Fig. 3b

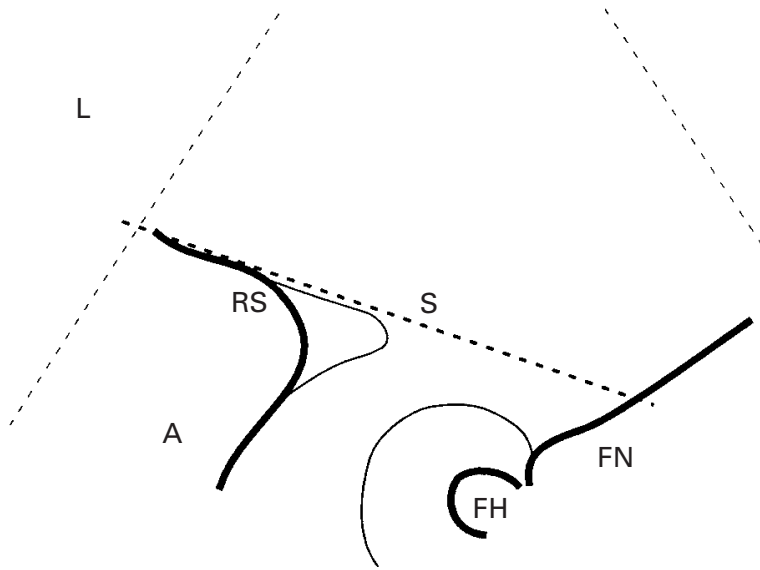

Fig. 3d

Ultrasound images of the same girl as in Figure 1, obtained with the patient supine using the method described in Figure 2. Figures 3a and 3b: the right side shows a full reduction. Figures $3 \mathrm{c}$ and $3 \mathrm{~d}$ : the left side shows a posterior dislocation.

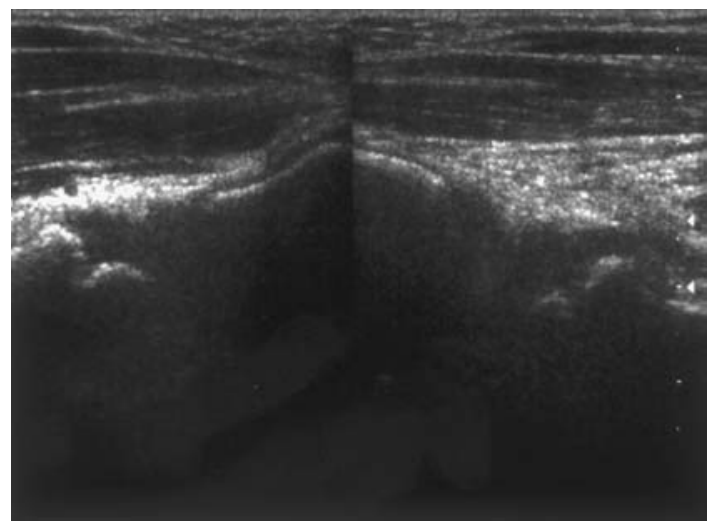

Fig. 4a

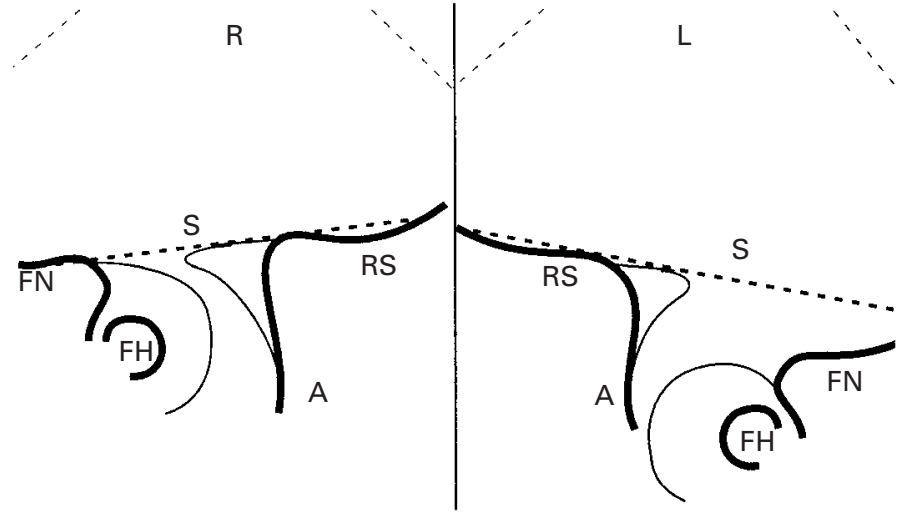

Fig. 4b

An ultrasound scan (a) with diagram (b) in the same two-year-old girl showing dual-mode images of hips after reduction for dislocation in developmental dysplasia of the hip (L, left; R, right; RS, ramus superior of the pubic bone; A, acetabulum; FH, femoral head; FN, femoral neck; s, Shenton line modified according to Smith et $\mathrm{al}^{3}$ ) 
in a spica cast, was used as a control in all patients. There is a learning curve to the use of ultrasound. The examiner must be familiar with the anatomical landmarks to obtain a proper scanning plane. The superior ramus of the pubis, the acetabulum, the femoral head and neck should be seen in one plane. First, the superior ramus should be identified, then, by moving laterally, the acetabulum and finally, by rotating the probe, the femoral head and femoral neck. The size of the perineal opening in the spica cast is important. It should be large enough to allow the ultrasound probe to be placed easily in the groin. If the perineal opening is too small, the investigator will not be able to see the landmarks in one plane. If we exclude the first two unsatisfactory ultrasound investigations, the remaining $16 \mathrm{CT}$ and ultrasound scans showed the same results. The expected interference from air in the soft tissues did not prevent adequate ultrasound examination after open reduction. As with CT it is possible to compare left and right ultrasound images if the ultrasound machine is switched to a 'dualimage mode' (Fig. 4).

Ultrasound therefore proved to be a reliable method of checking the reduction after closed or open reduction in a spica cast. It is now the method of choice for imaging in our clinic and CT is only used in specific circumstances such as when the ultrasound scan is unsatisfactory.
No benefits in any form have been received or will be received from a commercial party related directly or indirectly to the subject of this article.

\section{References}

1. Toby EB, Koman LA, Bechtold RE, Nicastro JN. Postoperative computerised tomographic evaluation of congenital hip dislocation. $J$ Pediatr Orthop 1987;7:667-70.

2. Hernandez RJ. Concentric reduction of the dislocated hip: computedtomographic evaluation. Radiology 1984;150:266-8.

3. Smith BG, Kasser JR, Hey LA, Jaramillo D, Millis MB. Postreduction computed tomography in developmental dislocation of the hip: part I: analysis of measurement reliability. J Pediatr Orthop 1997; 17:626-30.

4. Stanton RP, Capecci R. Computed tomography for early evaluation of developmental dysplasia of the hip. J Pediatr Orthop 1992;12:727-30.

5. Farber JM. A helpful radiographic sign in $\mathrm{CDH}$. Orthopedics 1992;15:1072-4.

6. Boal DK, Schwenkter EP. The infant hip: assessment with real-time US. Radiology 1985; 157:667-72.

7. Boal DK, Schwentker EP. Assessment of congenital hip dislocation with real-time ultrasound: a pictorial essay. Clin Imaging 1991;15:77-90.

8. Graf R. The sonographic evaluation of hip dysplasia using convexity diagnosis. Z Orthop Ihre Grenzgeb 1983;121:693-702.

9. Grissom LE, Harcke HT, Kumar SJ, Bassett GS, MacEwen ED. Ultrasound evaluation of hip position in the Pavlik harness. $J$ Ultrasound Med 1988;7:1-6.

10. Morin C, Harcke HT, MacEwen GD. The infant hip: real-time US assessment of acetabular development. Radiology 1985;157:673-7.

11. Novick G, Ghelman B, Schneider M. Sonography of the neonatal and infant hip. AJR Am J Roentgenol 1983;141:639-45.

12. Suzuki S. Ultrasound and the Pavlik harness in CDH.J Bone Joint Surg [Br] 1993;75-B:483-7. 